\title{
DOCUMENTATION
}

\section{Les grands Aménagements hydrauliques transalpins : L'Aménagement Piave - Lac de Sainte-Croix-Livenza.}

\author{
Por J. BOUDET. Ingénieur A. et M., Membre de l" "Ass. Elettrotecnica Ilaliana" \\ et de la "Société des Ingénieurs Civils de France"
}

\section{I. - Considérations générales. - Programme d'Ensemble}

La région du Lac de Sainte-Croix, située en Vénétie, entre les deux fleuves còtiers Piave et Livenza - et bien connue des touristes - quoique d'altitude modeste, présente un ensemble de conditions topographiques et orographiques particulièrement favorables à la réalisation d'importants. aménagements hydrauliques et qui ne pouvait manquer d'être avantageusement exploité, passionnant depuis longtemps techniciens et hommes d'affaires.

Etudié depuis une quinzaine d'années, un premier projet ayant pour but d'utiliser le lac de Sainte-Croix comme bassin d'accumnlation susceptible d'alimenter une série d'installations hydroćlectriques, fut réalisé en 1910 par la "Société italienne pour l'utilisation des forces hydrauliques de la Vénétie n (Sociétà Adriatica et Cellina) (1). Les résultats obtenus furent satisfaisants au point d'inciter la Société à étudier presque immédiatement des extensions susceptibles d'élargir dans une très grande mesure son exploitation; et c'est des études faites autour de ce projet d'extension que sortit, peu avant la guerre, le nouveau projet, à la vérité génial et considéré à cette époque comme très hardi, de détourner dans le lac de Sainte-Croix une partie des eaux de la Piave, de facon qu'en relation avec l'augmentation de l'accumulation de réserves en découlant, la puissance initiale des installations en soit quadruplée...

Ajournés, comme tant d'autres, du fait de la guerre et de l'invasion, les travaux purent néanmoins être entrepris peu après la signature de la paix, grâce à la ténacité de la Société, qui eut à surmonter les difficultés de tous ordres inhérentes aux perturbations économiques de l'après-guerre, jointes à de non moins sérieuses difficultés administratives et techniques; poussés activement, ils sont sur le point d'être terminés.

L'ensemble des anciennes et des nouvelles installations représente un exemple typique, grandiose même de l'utilisation rationnelle d'un aménagement hydraulique adapté aux circonstances locales, et mérite d'être connu, au moins dans les grandes lignes. Nous en résumerons ci-après les caractéristiques principales, d'après la description récemment publiée par M. N. Ratti dans L'Industria (2).

La région ainsi mise en valeur est située entre le Cadore et Trévise et les installations en question s'échelonnent sur une quarantaine de kilomètres entre Ponte delle Alpi sur la Piave (altitude $390 \mathrm{~m}$.), le lac de Sainte-Croix $(380 \mathrm{~m}$.), la vallée du Meschio, les lacs Mort $(275 \mathrm{~m}$.), de Negrisola $(160 \mathrm{~m}$.), et Fia, sur la Livenza (fig. 1.).

\section{II. - LES ANCIENNES INSTALtations}

L'ancien aménagement (fig. 2) est basé presque exclusivement sur la vidange du lac de Sainte-Croix, pour une capacité d'environ 40 millions de mètres cubes, étant donné que ce lac ne reçoit aucun affuent important. La dérivation consiste essentiellement en une galerie sous pression capable d'un débit maximum de $20 \mathrm{~m}^{3-}$ seconde débouchant dans la vallée du Meschio et alimentant Ia Centrale de Fadalto $(15.000 \mathrm{kw})$ où sont installés trois groupes de

(l) La chute du Cellina était utilisée dans la Centrale de Malvisio : débit $15 \mathrm{~m}^{3}$-seconde; chute $58^{\mathrm{m}}, 50$; puissance $6.000 \mathrm{kw}$.

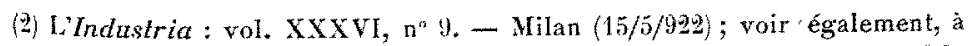
ce sujet, lEleltrotecnica, vol. IX, $\mathrm{n}^{\circ} 15$ : "Nuovi Implanti Idroelettrioci in corso di esecuzione . - Milano : Maggio 29.
4.500 HP et un groupe de $8.000 \mathrm{HP}$, fonctionnant sous une chute moyenne de 95 mètres.

Les eaux de fuite de cette première centrale, recueillies dans le lac Mort, qui sert de réservoir de compensation, alimentent une seconde centrale, dite de Nove $(12.000 \mathrm{~K} w$.$) , siluée à l'aval immé-$ diat du lac et utilisant, elle aussi, une chute moyenne de 95 mètres dans 2 groupes de $4.500 \mathrm{HP}$ et un de 8.000 -ce dernier installé depuis la Victoire.

Enfin, en aval de Nove, est établie la troisième centrale, dite de San-Floriano, écuipée, pour l'instant, avec seulement deux groupes de $1.400 \mathrm{HP}$ fonctionnant sous une chute moyenne de $14 \mathrm{~m}$, auxquels viendront s'ajouter prochainement deux autres unités de même puissance.

\section{III. - LES NOUVELLTS INSTALLATHONS}

Le nonvel aménagement (lig. 3) se développe parallèlement au premier, avec la seule différence, du resle capilale, que le lac de Sainte-Croix, dont la capacité d'accumulation est portée à 120 mill. lions de mètres cubes, est alimenté par les eaux de la plave au moyen d'un nouveau canal ayant sa prise sur ce fleuve, en amont de Ponte delle Alpi (altitude $385 \mathrm{~m}$.)

La nouvelle galerie sous pression qui alimente la nouvelle Centrale de Fadalto est, en conséquence, établie pour un débit de $100 \mathrm{~m} 3$-seconde, et cette nouvelle Centrale, située tout à colte de l'ancienne, est établie pour reccvoir jusqu'à 6 groupes générateurs de 24.000 HP, alimentés chacun par une conduite forcée de $2 \mathrm{~m}_{4} 60$ de diamètre et absorbant environ $20 \mathrm{~m} 3$ sous une chute variable de 84 à $110 \mathrm{~m}$.

Par analogie et symétrie, à côté de l'ancienne Centrale de Nove, sera construite une nouvelle usine comportant également 5 groupes de 24.000 HP. absorbant le même débit sous la même chute moyenne : 96 mètres.

Des eaux de fuite de Nove, une partie seulement - juscqu'd concurrence d'un débít maxímum de 45 m3-seconde - sera utilisée par les extensions prévues pour la Centrale de San Floriano (4 groupes de $1.000 \mathrm{KW}$, sous $14 \mathrm{~m}$. de chute), et le reste dévic, jusqu'au droit de Fia, sur le fleuve livenza (où sera creée une nouvelle Centrale - dite de la Livenza - que doivent éfuiper trois groupes de 24.000 HP fonctionnant sous chute de $124 \mathrm{~m}$ ), atl moyen d'un canal à écoulement libre qui, indépendamment de son utilisation industrielle, doit également servir, pour usages agricoles, aux fins d'irrigation des terrains situes sur son parcouts; c'est, du reste, dans ce but, qu'aux environs de Casteletto seront déviés, dn canal principal ci-dessus, $12 \mathrm{~m}^{3}$-seconde, destinés aux irrigations d'aval et quí, avant d'être livrés à l'agriculture dans son canal situé $60 \mathrm{~m}$. plus bas, utiliseront industriellement cette chute de $60 \mathrm{~m}$. dans une Centrale complementaire sise en cet endroit et équipée avec 2 groupes de $1.000 \mathrm{HP}$. Les travaux de cette petite Centrale sont actuellement poussés avec une grande activite, en vue de sa mise en service dès l'antomne prochain.

Lorsque l'ensemble des travaux sommairement esquisssés cidessus sera terminé, la puissance totale disponible, dont le maximum dépassera $250.000 \mathrm{KW}$, avec une moyenne régularisé, permanente, de plus de $80.000 \mathrm{KW}$ permettra d'assurer une prow duction d'au moins 650.000 .000 de K wh.

\section{IV. - Observatrons constructrves}

La réalisation de la première étape de ce vaste programme, commencée par la Nouvelle Centrale de Fadalto, au cours des annés 1920-1921 prete a diverses observations, da reste aussi heureuses qu'originales. On peut dire, par exemple, qu'elle eut deja, avant 
son achèvement, une répercussion très heureuse sur l'exploitation de la Société, car, pendant l'étiage exceptionnel de l'hiver clernier, le service de distribution, fort compromis sur tous ses voisins, put être maintenu sur le réseau vénitien sans trop de réduction, grâce à une installation provisoire avec pompes temps très anciens du fleuve sacré, qui, d'après Longarone, au lieu de décrire l'ample courbe actuelle touchant Belluno et les environs de Feltre, pour déboucher dans les plaines trévisaines au nord du Montello, effectuait un parcours direct, nord-sud, selon la ligne Farra d'Alpago - Serravalle - Vittorio Veneto.

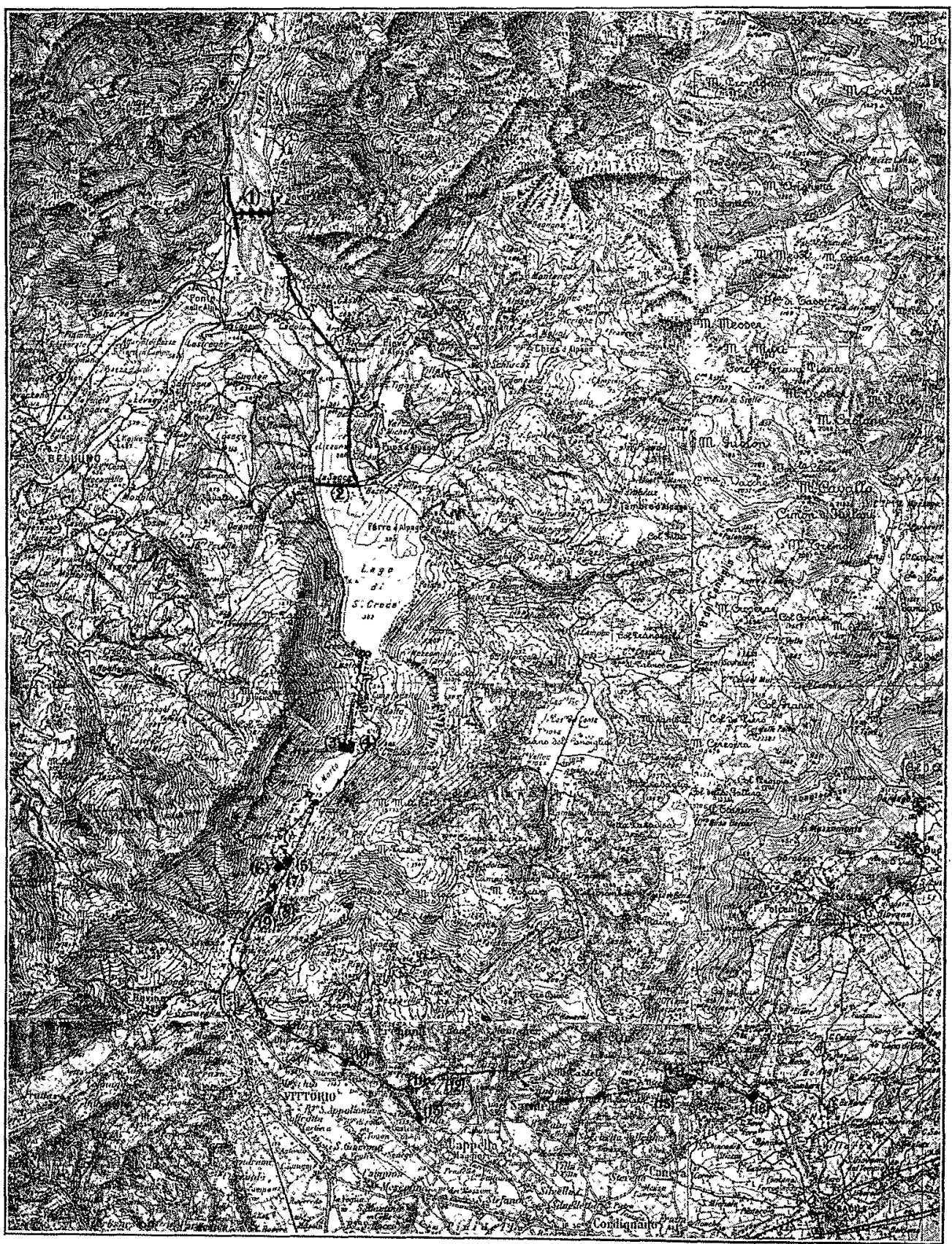

Fig. 1. - Carte générale de P'Aménagement Piave-Livenza.

\section{LEGGENDE}

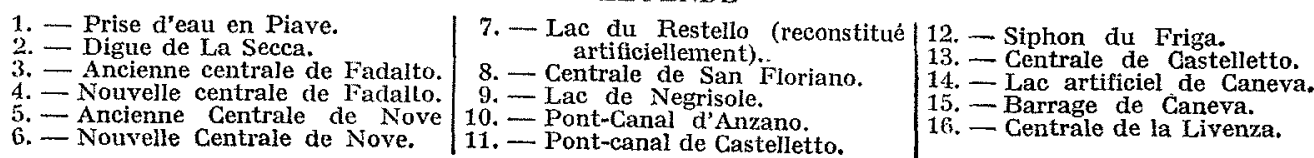

TABLEAU Synoptigue de L'AMÉnagement

\begin{tabular}{|c|c|c|}
\hline Etapes de réalisation & 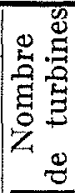 & $\begin{array}{l}\text { Puissance } \\
\text { totale }\end{array}$ \\
\hline
\end{tabular}

\section{I. - Chutes de Fadalto}

Installations réalisées..... $4 \mid 22.000 \mathrm{HP}$ Installations en construction $\quad 2 \quad 48.000-$ \begin{tabular}{l|l|l} 
Installations projetées..... & 4 & 96.000 \\
\hline
\end{tabular} \begin{tabular}{l|l} 
Total......... & $10 \quad 166.000-$ \\
\hline
\end{tabular} II. - Chutes de Nove

Installations réalisées..... $3|3| 17.000 \mathrm{HP}$

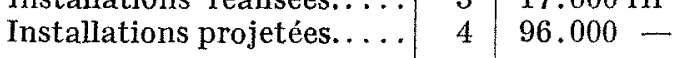
Total......... $7: \begin{array}{c:c} & 713.000 \mathrm{HP}\end{array}$

\section{III. - Chutes de San-Floriano}

Installations réalisées.... $1 / 1.660 \mathrm{HP}$ Installations projetées.... 2 2 $3.340 \ldots$ \begin{tabular}{l|l} 
Total.......... & 3 \\
\hline $5.000 \mathrm{HP}$
\end{tabular}

$$
\text { IV. - Chutes de la Livenza }
$$

\begin{tabular}{l|l|l} 
Installations en construction & 3 & $72.000 \mathrm{HP}$
\end{tabular}

Puissance totale obtenue après réalisation complète de l'aménagement général ......... $356.000 \mathrm{HP}$ élévatoires et canal en bois, au moyen desquels l'eau de la Piave était refoulée dans le lac de Sainte-Croix, faisant ainsi bẹnéficier les anciennes installations des dispositions maxima prévues pour le fonctionnement des nouvelles et réalisées par anticipation sur leur mise en service.

Une autre remarque, d'ordre hydrographique, qui n'est pas non plus sans intérét, découle de l'exécution même des projets que nous venons d'exposer; la partie des eaux de la Piave détournée de son cours actuel sur le Meschio et la Livenza reconstitue le régime des
V. - Equipement des Úsines

Les nouvelles Turbines "Rrva" DE $24.000 \mathrm{HP}$.

Les turbines fonctionnant dans les centrales de l'ancien aménagement, aussi bien que celles destinées aux nouvelles installations sortent toutes des ateliers de la maison "Costruzioni Meccaniche Riva ", de Milan. Parmi ces engins, les grosses unités de 24.000 HP, destinées à la nouvelle centrale de Fadalto, récemment terminées, et dont un spécimen fut exposé à la dernière Foire de Milan avec 


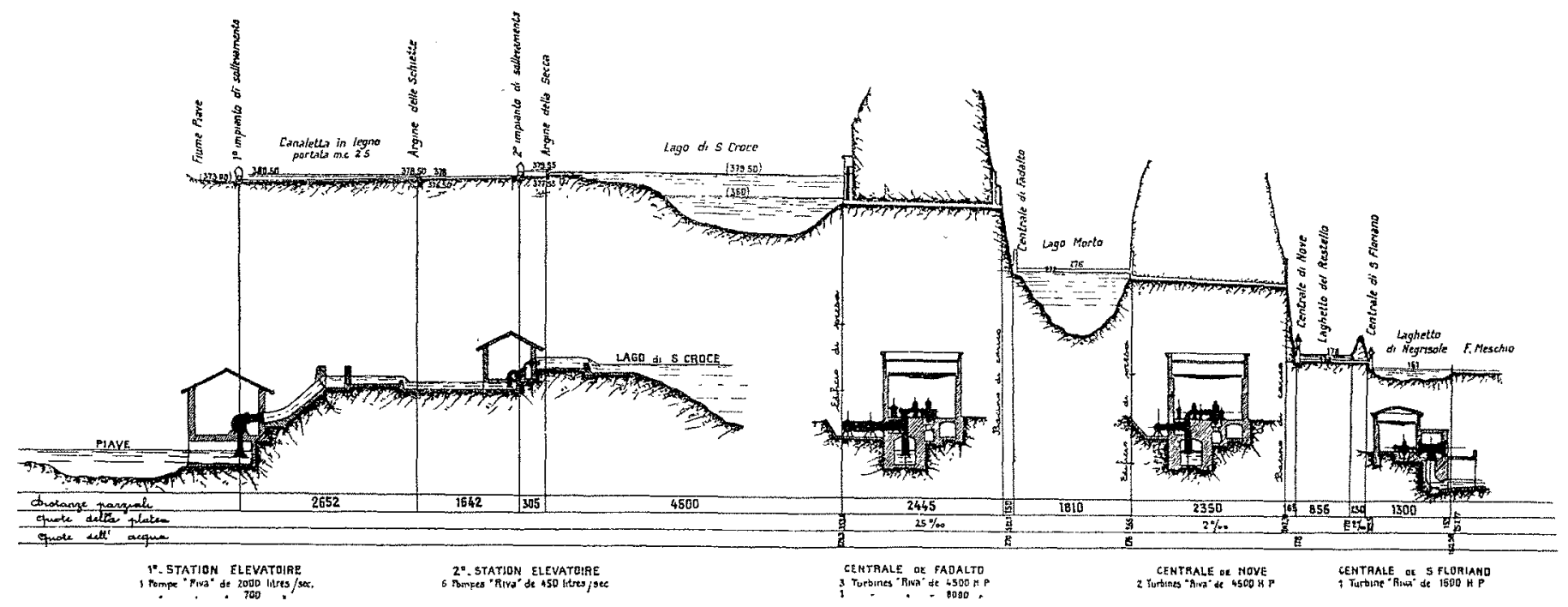

Fig, 2. - Profil en long de l'aménagenent initial : $(\mathrm{P}=41.600 \mathrm{HP})$.

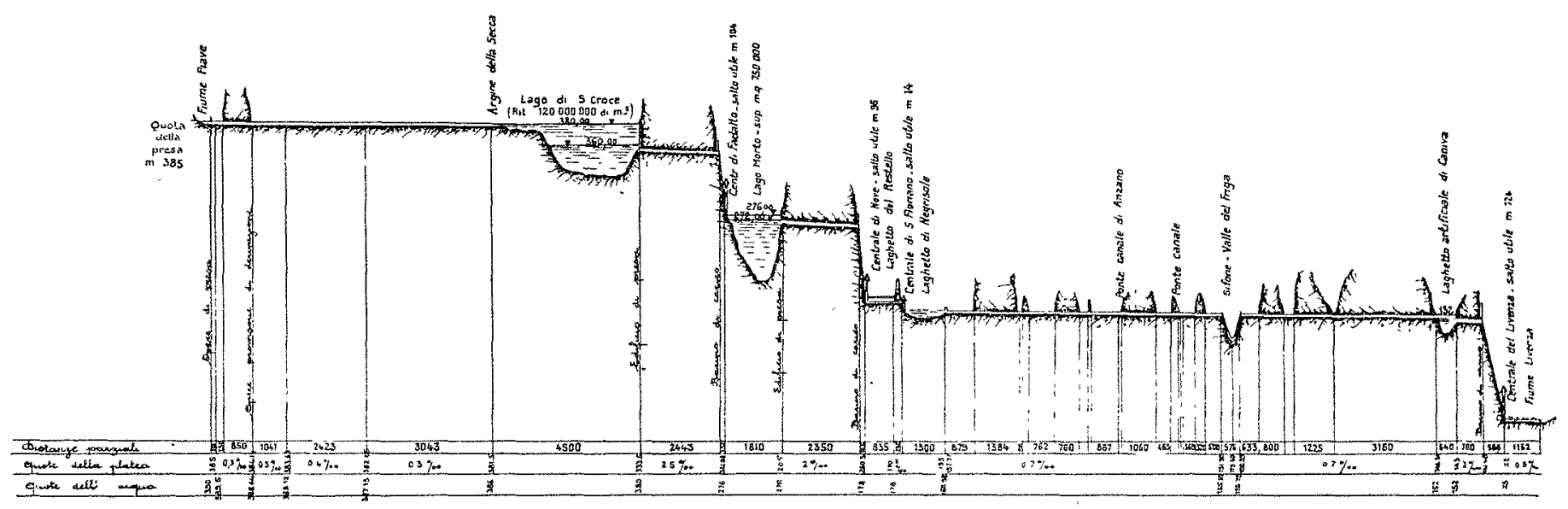

Fig. 3. - Profil en long dn nowvel aménagement, prévu pour une puissance tolale de 315.400 IIP., après achèvement; en cours de réalisation immédiate pour $52.000 \mathrm{HP}$. (1 ${ }^{\text {re }}$ étape du programme d’aménagement général).

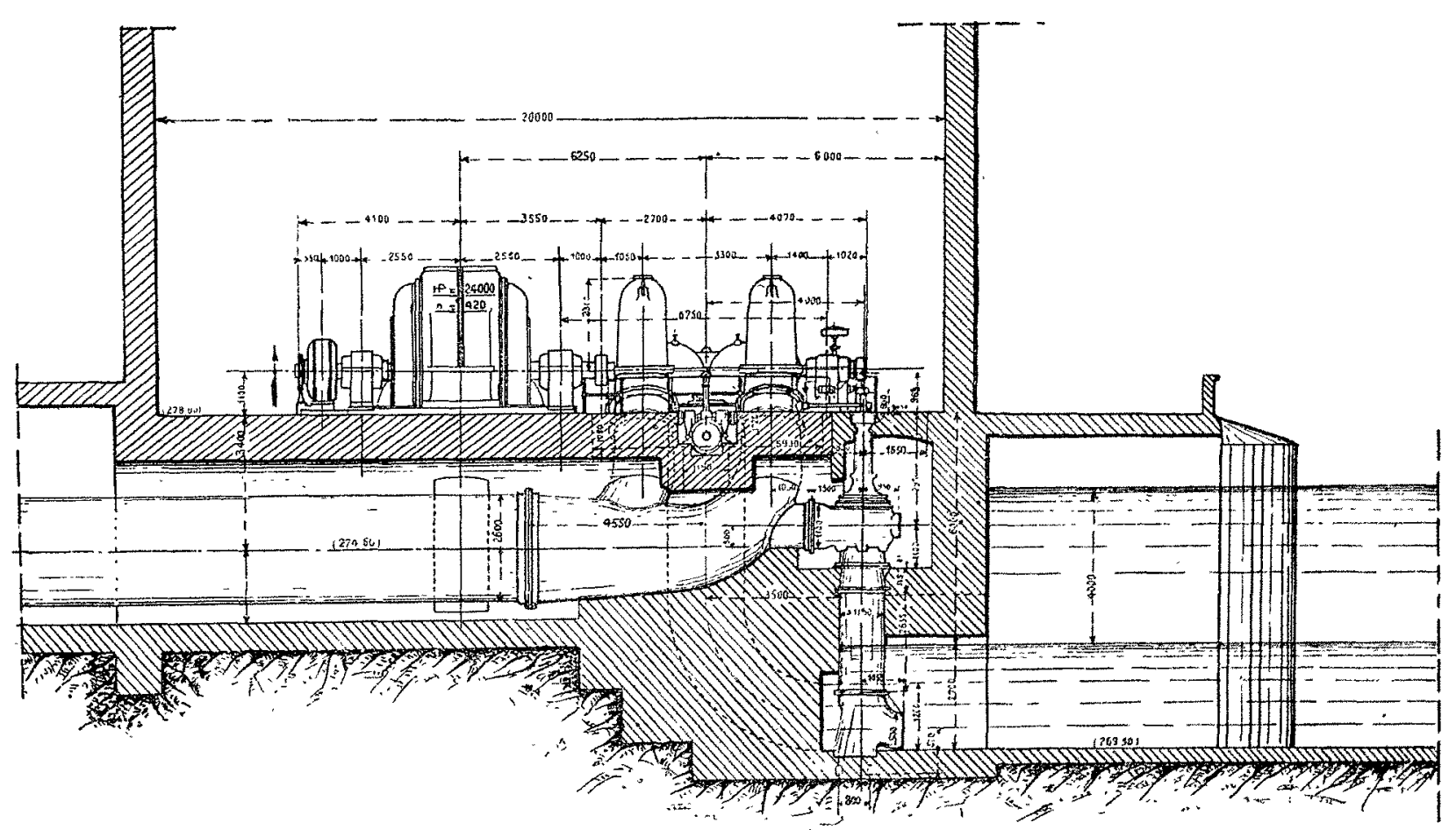

Fig. 4. - La nouvelle centrale de Fadalto : implantation des groupes turbo-dynamos atilisant les nouvelles turbines "Riva", de 24.000 IIP. 
un vif succès, méritenl une mention spéciale, aussi bien pour leurs dimensions que pour leurs caractéristiques spéciales qui réalisent les tout derniers perfectionnements de la mécanique appliquée à la construction des unités de grande puissance (Fig. 4).

Elles sont du type Francis, à 2 spirales et décharge -centrale raccordée avec un tube d'aspiration en béton : un tube à 2 branches reliant les spirales à la conduite forcée, avec interposition de 2 robustes vannes à papillon actionnées simultanément par un unique servo-moteur hydraulique. Le tube d'alimentation est situé audessous du plancher de l'usine. Les spirales, cui sont en fonte, présentent une section circulaire et sont construites en deux pièces superposées pour en faciliter le transport et le montage : elles sont éprouvées hydrauliquement à 17 atmosphères.

Les roues mobiles, en bronze, sont montées sur un arbre en acier avec flasques fondues pour l'accouplement avec l'alternateur. Les distribuleurs, à aubes Lournantes en acier avec commande par chaîne extérieure, peuvent être actionnés à la main, au moyen rl'un servo-moteur hydraulique, el automatiquement par un régulateur autonome à pression d'huile, d'un système particulier à la maison "Riva".

Chaque turbine est munie d'une grande vanne synchrone capable d'admettre plus de $16 \mathrm{~m} 3$ par seconde et pourvue d'un dispositif de fermeture automatique fonctionnant comme régulateur de pression. Sa mise en marche, de même que la mise en parallèle et la régulation de la charge s'effectuent depuis le tableau au moyen de commandes à distance appropriées.

Les alternateurs, accouplés aux turbines par manchon élastique, sont ćtablis pour produire du courant triphasé à 42 périodes, sous une tension de $6000 / 6600$ volts, élevée à 55.000 et 110.000 volts pour le transport.

\section{L'Électrification des Chemins de Fer en Italie.}

Après un bref historique et un rappel des premiers essais de traction électrique effectués il y a 21-22 ans sur les lignes de Monza et Varèze, l'auteur expose l'évolution et l'état actuel de la question, ce qui est fait, ce qui est prévu, les controverses qui se sont élevées dans le domaine technique et les critiques, aussi nombreuses que justifiées, adressées au projet d'électrification générale du Gouvernement, qui semble orienter assez mal la politique générale d'électrification de la péninsule, malgré tout l'intérêt qui s'attache pour elle à une libération aussi complète que possible, de l'autocratisme des ploutocrates charbonniers.

Il termine cette étude, aussi bien conduite que documentée en posant une question que d'aucums affirment angoissanle : "Quel intérêt présenterait la transformation - si onéreuse - de la traction sur les chemins de fer, le jour où le charbon baisserait de prix ? "et conclut que, de longtemps encore, le combustible n'atteindra: pas de cours assez bas pour pouvoir concurrencer avantageusement la traction électrique, avec laquelle les dépenses d'exploitation n'atteignent guère que $40 \%$ de celles que nécessite la traction à vapeur (statistiques de 1918 : pour $1000 \mathrm{t}$. transportées, 7,04 contre 17,16$)$.

Monitore Tecnico, $10-20$ avril 1922.

J. B.

\section{Rôle des Réservoirs dans les Aménagements hydrauliques.}

Quoique orientée et présentée, du reste, au seul point de vue nalional allemand, cette étude est pleine d'enseignement d'ordre général dont de nombreux techniciens pourront tirer profit. L'auteur y donne des tableaux et graphiques de débits pour les fleuves et rivières les plus importants et y expose (avec, à l'appui, de nombreux exemples de barrages-réservoirs en exploitation, et dont on connaît l'influence sur la marche des usines annexées) les régimes-limites pour lesquels il y a avantage à créer des réserves, quels sont les endroits et les conditions les plus favorables à la création des retenues, enfin quels gains on peut escomp- ter de cette création ; de ces donnćes il tire des conclusions tendant à l'établissement de théories entièrement nouvelles sur la régula. risation des cours d'eau "sauvages".

Par ailleurs, déplorant l'insuffisance de l'énergie hydraulique pour faire face aux besoins de l'industrie de son pays - (qui n'est du reste point le seul dans ce cas) - il envisage entre l'aménagement de cette énergie et l'utilisation de l'énergie thermique disponible une connexion étroite, comme seule capable de résoudre le problème de l'énergie en général.

Zeits. des Ver. Deutcher Ing., 29 avril au 13 mai.

\section{L'Aménagement de la haute vallée du Bergell et du lac des Sils (Suisse).}

Exposé très inléressant des difficultés d'un programme d'aménagement datant d'avant la guerre et à la réalisation duquel s'étaient opposés certains intérêts locaux ou régionaux, en particulier le tourisme, pour la protection des sites des Sils et de la Maloja, très fréquentés l'été, et l'agriculture, à cause des importantes variations que prévoyait le projet initial dans le niveau du lac des Sils. L'auteur démontre que les intérêts en présence n'étaient pas inconciliables et que le nouveau projet les satisfait tous.

Ce nouveau projet, actuellement en instance en vue d'une exécution prochaine et rapide, utilise une dénivellation de près de $1100 \mathrm{~m}$. que présente la haute vallée du Bergell sur un parcours de seulement $18 \mathrm{~km}$, entre le col de la Maloja et la frontière ilalienne, au moyen de deux chutes construites en série dont l'une utilise les eaux du lac des Sils (alt. $1800 \mathrm{~m}$.) et d'un autre, plus petit (situé à $2.400 \mathrm{~m}$.), sous $710 \mathrm{~m}$. de chute, dans une centrale située à Vicosoprano; et l'autre, sise à Castasegna, utilise, sous 390 mètres, les eaux de fuite de la première. Les équipements moyens prévus pour ces usines sont de 80.000 et $9.000 \mathrm{HP}$; la production moyenne annuelle escomptée est de l'ordre de 290 millions de Kwh.

Schw. Bzg. 1er mars 1922.

J. B.

\section{Un nouveau système de Joint assurant l'étanchéité des Conduites en Béton armé.}

Dans cette intéressante étude, l'auteur présente un système de joint réalisé par l'association de la fonte et du béton, dans le but d'assurer aux conduites en béton armé l'étanchéité qui leur fait trop souvent défaut.

Ce joint comporte deux manchons pouvant pénétrer l'un dans l'autre, fixés dans l'épaisseur des éléments de conduite et reliés i leurs armatures. Le manchon mâle porte un épaulement contre lequel on place un tuyau à gaz de grosseur appropriée, dont l'intérieur est bourré de filasse. Le manchon femelle est à section intérieure légèrement tronconique; de sorte qu'en l'enfilant par force sur son correspondant mâle, le tuyau en plomb s'aplatit progressivement jusqu'à s'écraser et constitue un joint particulièrement étanche, qu'on complète ensuite en terminant le bétonnage du bout femelle, demeuré inachevé pendant l'opération d'emboîtement des deux troncons.

Ce système a été appliqué récemment en Amérique avec plein succès pour des conduites de 0,90 à $1 \mathrm{~m}$. 20 de diam. coulées sur place dans des coffrages métalliques préalablement huilés, et faites avec du béton dont la fluidité était augmentée par l'adjonction d'argile au mélange, dans la proportion de $2 \%$. L'auteur recommande particulièrement ces dispositions spéciales comme autant de facteurs de succès.

Engineer, 14 mars 1922.

J. B. 